\title{
AN ENHANCED RAILWAYS CONTROL OFFICE APPLICATION IMPLEMENTATION IN CLOUD ENVIRONMENT
}

\author{
M. Manimekalai ${ }^{1}$, R. Raghuraman ${ }^{2}$ \\ ${ }^{I}$ Director and Head, Department of Computer Applications, Shrimati Indhira Gandhi College, Tiruchirappalli, \\ Tamilnadu, India \\ ${ }^{2}$ Principal, Zonal Railway Training Institute, Southern Railway, Tiruchirappalli, Tamilnadu, India
}

\begin{abstract}
As a result of continuous advancement of research and technology, travelling from one to place to another has become affordable and more at ease. There are opportunities which exist even for travel from Earth to Mars and other planets. The developments in the field of transportation have made a colossal impact in travelling for the passengers and other customers who rely on mass transportation of their goods. Many of the travel surveys reveal vital information about trips performed by people like origin, destination, mode, duration, distance and purpose of trips, as well as socio-economic and demographic data for investigation. This has made it possible to provide the best possible mixture of Fixed facilities, Flow entities, Operational plans, Storage facilities and Control systems wherein it allows the people and goods to trounce the abrasion offered by geographical spaces in a competent, secured and more affordable manner to attain the time and place benefit for the desired activity. The role of Indian Railways in maintaining train operations is effectively done by the Control Rooms in each of the seventy seven divisional/ area control offices. These Control Rooms are the nerve centre of the divisional operations. The effectiveness in maintaining the train schedules lies with the staff who are in charge of the Control Room operations. The Control Office never shuts down its operations and works $24 X 7$ to monitor and maintain fluidity of operations over its jurisdiction. In the recent days, train movements are supervised and captured in real time with the use of Control Office Application (COA) for effective monitoring of both scheduled and unscheduled trains. With the deployment of COA, a scheduled train can be started from one terminal to another by plotting the graph through getting information about the train from the stations manually, i.e., the information will be collected by a Controller on duty and the details collected include station name, train number, arrival time and departure time. This captured date is used for plotting the graph showing the movement of the train in the direction of its run and it also aids in fixing crossing or precedence of two or more trains at the best location based on conflict protocols. In this paper, we propose a new algorithm for voice-to-text conversion in mechanizing the scheme of information capture in the COA wherein the system would still capture the information from the station even when the Controller (human agent) is not available or when the inputs are received from more than one station. The proposed structure gives an opportunity to help us to overcome the human dependence and related errors in collection of information from the field.
\end{abstract}

In the automation mode, when the human agent is physically not available, he will have the facility to switch on to the automated mode through which the information from the station can be collected as voice input, recorded and then converted into the text using the proposed algorithm. This would enable the application to use the data in an uninterrupted fashion for plotting the further movement of the train and also use the data for advance plotting in a more realistic fashion. The cloud is a set of hardware, networks, storage, services, and interfaces that facilitate the deliverance of computing as a service. In this type of cloud we are implementing our COA application software. It reduces the cost of hardware, hardware problems, servers.

Keywords: COA, FOIS, CRIS, NTES, RAS, MIS

\section{INTRODUCTION}

Each and every application necessitates the Controller to enter the data related to the train operations in the COA as soon as it is received from the stations. The graphical chart provide in the COA [1] provides visual information about the running of the rain in a section (a portion of the divisional network) and also facilitates plotting of the advance forecast based on various operational parameters. This enables the train to be virtually handed over to the neighbouring division as per its physical movement and provide seamless flow of information.
At present, the COA has been structured in such a way there can be seamless exchange of data with any other application. Some of the key applications like National Train Enquiry System (NTES) [2], Punctuality Analysis Module (PAM) of Integrated Coaching Management System [1], and Freight Operations Information System (FOIS) [3] [4] are all integrated through an Enterprise Application Integration Software with COA. As a result of constant development in technology, CRIS (Centre for Railway Information Systems [5], Indian railways IT wing, has created an improved interface at www.trainenquiry.com for dissemination of information to public. NTES (The National Train Enquiry System) [2] is a backend system which 
facilitates information about running of trains on real time basis and provides the same through different interfaces like nationwide unique number 139, mobile interfaces, Website and face to face enquiry etc.

The website's new interface was opened to the public from 6th September 2013. The new interface, which provides fast and easy access to key information for passengers, has been chiefly valued for its information richness, speed and simple interface and richness of information through smooth user experience. To further make it widely popular, a light weight mobile compatible web interface has also been introduced to provide information through various types of enquiries via mobile internet while on move. The Classic and Modern flavor of mobile web edition have also been offered to support Generic mobiles and Smart phones.

The special feature of Web NTES is "Spot your train" which is endowed with information such as departure time of the train at the particular station, expected arrival time and current position of the train. In addition to this, the other unique features are 'information on rescheduled cancelled and diverted trains', 'live stations', 'trains between stations' and 'train schedule'. 'Live Station' query provides list of trains expected to arrive at/depart from any station in the next $2 / 4 / 6 / 8$ hours.

The algorithm and functional elements of the innovative method being proposed include data manipulation, human voice detection and speech recognition. In the fast growing robotic technology, looking for an opportunity in understanding the concepts in real time has become much easier. To provide the desired level of standard framework, libraries, support developers and tools for robots composition have become more accessible. They speed up the growth of the construction of stand-alone robots and an individual functional modules by recuperating their reusability and mutual connectivity. One step further in this direction, current research has persisted on the approach of Networked Robots, which surmounts the margins of standalone robots by having robots, environment sensors, and humans to correspond and assist through a network [2]. Initially, a team in Japan had proposed the concept of networked robots in the year 2002 [6]. Subsequently, numerous classifications have been proposed, including the one by the technical committee of IEEE Robotics and Automation Society (RAS) [7]. These networked robots have turned out to a tool for vigorous investigation in the field of robotics, and numerous projects have been taken up to prove how this concept can augment the ability of individual robotic services. These kind of techniques have amplified the capability of service robots to systematically support the routine activities [8]. In our regular life, it is rare to keep doing a same task at a given place. We usually, wake up in the morning, brush our teeth, wash our face and eat breakfast. In the daytime, we may go for grocery shopping or take a tour of a bookstore. Sometimes we may require some medical treatment at a clinic. After returning home, we mostly do our regular housework such as washing of clothes, preparation of dinner, and so on. But occasionally, we do take care of our pets or do gardening. These are some examples of regular activities in our daily life done sequentially under different contexts.

\section{COA IN RAILWAY OPERATIONS}

In the railway operations, the train movement is the primary function. The control chart forms the core part of train operation as it offers a visual tool for facilitating operational decisions. The main requirement of the Computerized Control Charting system is to handle high traffic volume over the Indian Railways in an efficient manner. The COA [9] is a mission critical application of the Indian Railways which runs on $24 \times 7$ basis. CRIS has developed the software for COA and rolled out the application all over the Indian Railways.

Among the various government sectors, Indian Railways is one of the most highly developed organizations with a pioneering and extensive IT environment. The historical moment began as a $33.6 \mathrm{~km}$ stretch of rail in 1853 and at present, IR has become the Asia's largest train network. Indian Railways operates a very large network wherein approximately 19,000 trains run daily on more than 104,000 kms of track spread over three different gauges. In 1986, the Ministry of Railways founded Centre for Railway Information Systems (CRIS) [5] [9], to be a parental organization for all computer activities of Indian Railways (IR) [9]. It is mainly a project oriented organization and accountable for building major computer systems for the Railways. Moreover, it integrates the effort of the Indian Railways to ensure standardization, affords high levels of expertise and gives the organization the litheness to continue the tempo with fast growing technology.

The Indian Government owns the Indian Railways which is responsible to transport more than 8.5 billion people and 1100 million metric tons of freight traffic [9] each year. The Railways are divided into different zones, divisions and each division is further divided into control boards of varying length. The broad structure of Indian Railways is made up of 16 zonal railways, which in turn are alienated into a total of 68 divisions. The core function of the Section controller of each Control board in each division is to ensure that the trains are run safely and on time. At the outset, controllers monitor and plan the train movements manually, which means that they have to spend majority of the time in undertaking mundane and repetitive tasks. As a result of this, the work of Section Controllers on busy boards has become very strenuous and restricts strategic planning [10].

The nature of train operations requires exact and precise operational strategies - which has necessitated the Indian Railways to switch over a client-server application for managing the movement of trains effectively [10]. The pilot project was tested out only in two Divisions to get wide ranging user feedback in improving the features in the application. However, this application needed an instantaneous refresh of the chart based on new inputs, which however affected the application performance and the refresh rate of the chart extending up to 50 seconds. 
However in the manual process, the controller needed to only change some parameters to derive at the result. The perfect approach needed to be oriented towards automatic train ordering, where the system exhibits all possible routes, facility like diversion, re-routing etc. The details regarding the Train Consist, Crew, BPC, Loco etc [10]. are used to describe about other related events. In addition, the chart illustrates line occupancy, caution orders, abnormal working, zoom facility, horizontal scrolling etc.

The other requirements of the chart include auto saving of the chart at pre-determined interval, printing of chart as an image. The MIS reports [11] are used to project the performance results related to Punctuality, Hours on Run, Interchange Speed Restrictions, Maintenance blocks, Unusual Occurrences, Equipment Failure, Loading/Unloading position etc. The Controller also has facility to add remarks and comments by using electronic pen. The other features include SMS alerts to concerned users for specific events. There are also features for electronic flow of information through COA from one division to the adjoining Division about the current running help us of trains and probable arrival at the interchange point.

The Advance plotting feature is predominantly dependent on parameters like speed restrictions, maintenance blocks, deduction of allowances (Engineering /Traffic), line occupation of stations, priority of trains, scheduled halts, absolute block check, unusual occurrences etc [12].

\section{CURRENT METHOD IN COA}

The existing method in COA as implemented over Indian Railways formulates the Controllers to collect data about the movement of trains from the stations assigned to a Control board. The COA application tracks on the n-tier architecture design [13] which consists of the following components: Presentation Layer, Business Layer and Data Access Layer Control View, Data-Entry and Cache.

\subsection{Presentation Layer}

The presentation layer has been divided as mentioned in the following components: Control View, Data-Entry and Cache. The presentation layer has been developed as a smart client solution with Click Once capabilities for zero-touch deployment.

\subsection{Control View}

This component is liable for depicting the control chart as a graphical user interface (UI). In addition, the controller fritters away most of the time in this view to plan the further movement of trains and to take firm decisions (such as stopping a goods train on a line at a station so that express trains can move ahead etc).

The user interface (UI) has options to identify the train types as (Express, freight, passenger), in distinguishing the upstream and downstream and illustrate this information through vector graphics support as part of Windows Vista and Microsoft .NET Framework 3.0 component. This kind of pictorial representation is called Windows Presentation Foundation (WPF) [14].

The advancement of the technology has opened up facilities for instantaneous rendering of chart which would help the controller in making decisions quickly and smartly on a high-traffic board. The COA gives an open view through the Windows Presentation Foundation, which affords new techniques for emerging applications, represent richer graphics and, more significantly, the facility to provide incremental refreshes to bring out real time data in graphical form at the shortest possible time.

\subsection{Data Entry}

The visual component provides for quick data entry by the controller in a most efficient way. The data captured include movement details, commentary on specific event relating to trains, lines used for receiving a specific train at a station etc. Moreover, it also facilitates information on abnormal working conditions, speed restrictions etc.

The major objective of the component is to capture the above information as quickly as possible to aid the controller in providing the graphical view to observe and make decisions about further movement of trains. The information captured on this screen is stored in a local database and also updated in the cache. Once the information is updated in the database as well as cache, the control view component is signalled through .NET mechanisms such as event and renders the chart afresh with new data.

\subsection{Business Layer}

The main process of COA is to logically encapsulate the structure of .NET components [15] which forms the business layer of the application. The Controller feeds the information in the presentation layer. The UI authenticates the information and transfers it to the business components for business validation and logic checks. The validated information is sent to the presentation layer for the controller to view the information in desired format.

\subsection{Data Access Layer}

The master data (referential) (e.g. board information, trains running on board etc.) is termed as reference information for describing the control chart and for making validated decisions. Since this information seldom changes, it is cached in memory, but when they do change, the cache is refreshed.

The controller or board specific data (e.g. movement information) is captured by the controller and logged into the transactional database. 


\subsection{Printing of Charts}

The COA provides facility for the control charts to be printed, for analysis, legal and archival purposes, in high resolution. The printing is done in XML Paper Specification (XPS) format [16] and open document format that uses Extensible Markup Language (XML) [17]. The charts can be viewed and analyzed in the browser, in high resolution as compared to most common jpeg format. This facilitates easy archival and can also be used as data source for future requirements.

\subsection{Forecasting Services}

A forecasting algorithm provides near comparable predictions based on high degree of computation at least time ranging between anywhere between a very few seconds to minutes of the CPU time. The forecasting service is run as a process through multiple application servers to reduce the time taken to render the forecast. Advanced Web services support in Windows Communication Foundation, a core component of the .NET Framework 3.0 provides secure, reliable, and transaction messaging along with interoperability. The service-oriented programming model of Windows Communication Foundation [18] is built on the Microsoft .NET Framework and simplifies development of connected systems.

Forecasting service tool is a Web service using WCF framework and listens on a TCP endpoint on the forecasting server [18]. The client connects to the service through WCF client proxy and sends the advanced forecasting request. The Server, upon receiving the request, can figure out whether a most recent forecast data is available for the board. Each service runs advanced forecasting algorithm for different boards continuously or runs in a pre-defined interval. After each computation, the result is updated in the database as well as in the cache.

\section{BENEFITS OF THE CURRENT METHOD IN}

\section{COA}

The Control Office Application provides a platform for betterment in the efficiency of train running operations, enforcing safety and ease at work. These benefits would get transformed into greater customer satisfaction, as well as increased throughput using the same rail infrastructure. This would pave way to increase in revenues and better utilization of assets over Indian Railways.

\subsection{Reduction of Charting Time from 20 Seconds to}

\section{Sub One Second}

The core function of the COA was to provide the controllers with a tool which simplifies their mundane work and give them scope for better decision making. . While the first version of COA automated the process, the second version of the application provided astonishing benefits thereby improving the acceptance level of staff over the Indian Railways [19].
"The controllers who are presently using the application, find a remarkable advancement as the data is rendered automatically on their screens giving a sub second response time which is impossible to match manually," comments Mr. Sanjaya Das, General Manager - Projects, CRIS.. He further adds, "It has also helped to alleviate pain points such as repetitive tasks, allowing the controllers to focus on other critical activities."

\section{PROBLEM IN THE CURRENT PROCESS OF}

\section{COA}

In the current scenario, the hardware requirement for implementation of the COA in every control office leads to increased initial cost and constant need for up gradation. In the existing method of capture of data from the stations and other activity centres, the human agent receives the communication from each station through the telephone.

There are atleast 20-30 stations under a Control Board and in some cases the quantum of traffic handled may warrant collection of data for multiple trains at the same time. There are also chances that the call from a station is missed or goes unnoticed during simultaneous attempt through omnibus telephone circuit.. In this kind of situation, a single or multiple error may crop up and result in negative impact or wrong input which would affect the forecasting service adversely.

There are also situations where the Controller goes to the Shift In-charge to take instructions for a given situation or goes to attend nature's call or for any other emergency requirements. In such kind of situations, the manual process doesn't provide an alternative for collection of data and there is more likelihood that some of the key inputs are not captured during such situations. A conceptual approach has paved way for introduction of an automatic mode i.e the voice inputs from the stations can be received by the system and converted into data string for storing in the database and further plotting of the graph governing movement of the trains. This automatic mode will be more effective and helpful and assist the Controller in effective capture of data which would form the key input and improve the performance standards of COA considerably.

\section{VOICE-TO-TEXT ALGORITHM FOR AUTOMATION MODE}

The proposed algorithm would facilitate sensing of non availability of the shift Controller or his/her not responding to a call by a station and enable recording of the voice input containing basic data like the station name, train number, arrival and/or departing time. This voice input would be converted into text (more specifically as a record set) and get stored in the database and the stored data used by the COA for rendering the chart based on the last updated input. The advantages of the proposed system over GPS mapping or other technologies like data logger or RFID is that the initial cost and recurring cost on the provision hardware at locations or on board the train would be brought almost to 
zero and thus highly cost saving. The following steps are included for the conversion of voice into to text.

Step 1: Take the call when the automatic mode is on activation.

Step 2: Get the inputs like station name, train number, and arrival and/or departure time from the operator of the station through telephonic call.

Step 3: The call attended through telephone is received by means of the receiver.

Step 4: The recognition of the speech i.e station name, train number, and arrival/departure time takes place.

Step 5: The captured speech inputs are checked with the defined semantics that exist in the dictionary.

Step 6: If the voice input matches the pattern, then it is converted into text using visual studio, SQ Lite and Microsoft speech recognition engine and stored in the database.

Step 7: Any exceptional data is stored in a separate error_log and resolved conveniently.

\section{IMPLEMENTATING THE PROPOSED COA METHOD THROUGH CLOUD COMPUTING}

The proposed software framework is planned to be implemented in the cloud environment to avoid the difficulty of the user who would be using the COA functional features.

The security $\operatorname{logs}$ for the access control and database is retained at the user level and managing logs are done by this software. The proposed changes in the software would provide two types of mode -Manual Mode and Automatic Mode.

The manual mode would require the Controller to collect the inputs from the station and enter the data in the system through defined user interface.

The automatic mode can be physically chosen by the Controller or auto-enabled if there is no responses from the Controller for a pre-defined period say 10 or 15 seconds. This shall be configurable.

This software can be implemented in the cloud as Software as a Service (SaaS) [20]. The relative advantages of Saas are listed below :-

- Software is managed from the central location.

- Software is delivered as a "One to many" model.

- Web access is enabled. No dedicated network is required.

- API (Application Programming Interface) is to be allowed for the integration of the different pieces of the software.

- The handling of the software patches and upgrades is not required to be undertaken by the user.

\section{RESULT DISCUSSION}

By implementing the proposed framework in the cloud computing with the intiation of automation mode, the following benefits can be achieved.

* High reliability with $99.9 \%$ of input capturing.

* Handle very high volume of traffic

* Provide relief to the Controller from mundane tasks

* Cost saving technology

* Easy adaptability

* Fulfill statutory requirements in capture and storing of data.

* Improved advance forecasting.

\section{CONCLUSION}

Software as a Service (SaaS) platform is not only a development platform but also a resources platform. In the scheme of SaaS, all data and software can be used as services. These services represent as API or as applications provided for users.

The development of a module in SaaS and under traditional environment are not very different. The key to success in the process is a paradigm shift from the established software development environment and adopting SaaS as a technology.

At present development in SaaS doesn't have any unification industrial standard There are a lot of works to be done in understanding the SaaS architecture. By implementing our software framework in the cloud, we can have distributed geographical agent centres; the usage of resource can be independent of the user of our software in the cloud. The software framework would also multiple application subsystems and there may not be any chance for agents- server problem or availability problem of the agents.

\section{REFERENCES}

[1]. "Monitoring Freight trains operations in Indian Railways", Management of Goods Trains in Indian Railways.

[2]. Sandhya Shama Rao, “Case Study - Tracking Solution for Indian Railways", Wipro Company.

[3]. "Chapter 2 - Freight Operations Information System in Indian Railways", Report No.8 of 2010-11(Railways) of Indian Railways.

[4]. Ms. Seema Agarwal, Ms. Ruchi Singhal, Ms. Rama Mittal, "Freight Operations Information System (Fois)An Advanced Architecture \& It's Future", Volume 2,Issue 2 :Page No.118-120, March - April (2013), International Journal of Latest Research in Science and Technology.

[5]. "Centre For Railway Information Systems", Report And Accounts for the Year Ending $31^{\text {st }}$ March, 2012.

[6]. Koji Kamei, Miki Sato, Shuichi Nishio, and Norihiro Hagita, "Cloud Networked Robotics".

[7]. Craig Schlenoff, "National Institute of Standards and Technology- IEEE Ontologies for Robotics and Automation". 
[8]. Marcus Mast, Michael Burmester, Birgit Graf, Florian Weisshardt, Georg Arbeiter, Michal Španěl, Zdeněk Materna, Pavel Smrž and Gernot Kronreif, "Design of the

Human-Robot Interaction for a Semi-Autonomous Service Robot to Assist Elderly People", Design of the Human-Robot Interaction, Springer International Publishing Switzerland 2015.

[9]. "Operating Manual for Indian Railway", Government of Indian Ministry of Railways (Railway Board).

[10]. "Control Office Application Functional Requirements Document", Part-4 of Tender Document of Indian Railway.

[11]. Shri Bibek Debroy, Shri K.M. Chandrasekhar, Shri Gurcharan Das, Shri Ravi Narain, Prof. Partha Mukhopadhyay, Shri Rajendra Kashyap, Shri Ajay Tyagi and Sh. Ajay Narayan Jha, "Interim Report of the Committee for Mobilization of Resources for Major Railway Projects and Restructuring of Railway Ministry and Railway Board", March 2015 Ministry of Railways Rail Bhavan New Delhi.

[12]. "Computerised Passenger Reservation System of the Indian Railways", Chapter 1 Computerised Passenger Reservation System of the Indian Railways.

[13]. Mumtaz AL-Mukhtar and Sarmad Hadi, "Developing a Three Tier Web Data Management Application for Higher Education Admission Environment", International Arab Journal of e-Technology, Volume 2 number 4, 2012.

[14]. Ian Griffiths, Chris Sells, "Programming Windows

Presentation Foundation".

[15]. Chris Anderson, "Essential Windows Presentation Foundation - Microsoft .Net Development Series".

[16]. ECMA Final Draft Standard, "Open XML Paper Specification".

[17]. Rami Alnaqeib, Fahad H.Alshammari, M.A.Zaidan, A.A.Zaidan, B.B.Zaidan, Zubaidah M.Hazza, "An Overview: Extensible Markup Language Technology", Journal of computing, Volume 2, Issue 6, June 2010.

[18]. Markus Stopper and Bernd Gastermann, "Serviceoriented Communication Concept based on WCF.NET for Industrial Applications", Proceeding of the International Multi Conference of Engineers and Computer Scientists 2010 Volume 3.

[19]. "Data Logger System", Efftronics- To Provide insight for Enhancing Wealth.

[20]. K.V. K Mahesh Kumar, "Software As A Service For Efficient Cloud Computing", IJRET: International Journal of Research in Engineering and Technology. 